\title{
NK and Th17 Cells in The Thymus of Myasthenia Gravis Patients
}

\begin{abstract}
Lala Mehdikhanova ${ }^{1}$, [MD] ORCID: 0000-0003-1239-8987

Can Ebru Bekircan - Kurt ${ }^{1}$, [MD]

ORCID: 0000-0003-2355-6979

Gurcan Tunali ${ }^{2}$, [MD]

ORCID: 0000-0001-8204-6819

Aslı Tuncer ${ }^{1},[\mathrm{MD}]$

ORCID: 0000-0001-9449-4483

Sevgen Önder $^{3}$, [MD]

ORCID: 0000-0002-5523-0669

Erdem Karabulut ${ }^{4}$, [MD]

ORCID: 0000-0002-7811-8215

Mustafa Yilmaz ${ }^{5},[\mathrm{MD}]$

ORCID: 0000-0002-0881-6747

Gunes Esendagli ${ }^{2}$, [MD]

ORCID: 0000-0003-4865-2377

Sevim Erdem-Ozdamar ${ }^{1}$, [MD]

ORCID: 0000-0001-9348-6457

${ }^{1}$ Hacettepe University Medical Faculty, Department of

Neurology, Ankara, Turkey

${ }^{2}$ Hacettepe University Cancer Institute, Department of Basic Oncology, Ankara, Turkey

${ }^{3}$ Hacettepe University Medical Faculty, Department of

\section{we A B T RACT Cen}

Objective: As a classical autoimmune disorder, anti-acetylcholine receptor antibody positive myasthenia gravis has an unconventional pathophysiology that involves thymus, the central organ for immune tolerance induction. Both natural cells and type 17 helper $\mathrm{T}$ (Th17) cells possess capacity to influence autoimmune inflammation. This study aims to determine the presence of Th17 and natural killer cells in the thymus from myasthenia gravis patients.

Materials and Methods: Thymectomy materials of myasthenia gravis patients and non-myasthenic controls were assessed by CD56, CD16, CD2, CD3, NKG2D, NKp46 and IL-23R flow cytometry and IL-23R, IL-21R, and ROR- $\gamma$ immunohistochemistry. Results: Even though natural killer cell infiltration was limited, the majority of these cells displayed activation markers NKG2D and NKp46. Expectedly, the amount of $\mathrm{CD} 2+$ lymphocytic cells were higher than $\mathrm{CD} 3+$ thymocytes in which a considerable percentage was carrying the receptor for IL-23 (IL-23R). In addition to IL-23R, IL21R, and ROR- $\gamma$ were also detected in myasthenic thymus as a marker related to Th17 cells. These Th17-related markers were reduced in thymoma compared to that of detected in thymic hyperplasia or the myasthenic thymus with normal histopathology. Conclusion: Both natural killer cells and Th17 cells are found in the myasthenic thymus indicating a possible cross-regulation between these cell types that may influence the course of autoimmune reactions.
\end{abstract}

Pathology, Ankara, Turkey

${ }^{4}$ Hacettepe University Medical Faculty, Department of

Biostatistics, Ankara, Turkey

${ }^{5}$ Hacettepe University Medical Faculty, Department of

Cardiovascular Surgery, Ankara, Turkey

Corresponding author: Can Ebru Bekircan-Kurt

Hacettepe University, School of Medicine, Neurology

Department, Neuromuscular Diseases Research Laboratory Sihhiye, Ankara /TURKEY

Phone: 903123051741

Fax: 903123093451

e-mail: canebru@yahoo.co.uk

Received: 16 July 2020, Accepted: 21 July 2020,

Published online: 30 September 2020

\section{INTRODUCTION}

Myasthenia gravis (MG) is a rarely encountered antibody-mediated and CD4+ helper T cell-dependent autoimmune disease of skeletal muscles. These antibodies affect neuromuscular junction generally by targeting acetylcholine receptors (AChRs) and lead to muscle weakness [1]. Although the etiology of autoimmune dysfunction in MG is not clear,
Key words: Myasthenia gravis; NK cells; thymus; Th17; IL23R. thymus abnormalities have been demonstrated to have an important role [2]. In MG, the thymus can be recognized with a neoplasia-like presentation which include tumor (thymoma) development and follicular hyperplasia that are characterized with the generation of B lymphocyte-rich germinal centers [3]. The presence of 
secondary lymphoid structures, B lymphocytes, and plasma cells in such an organ which is responsible of $T$ cell development and selection of non-autoreactive clones has been regarded as a major feature of MG immunopathology [4-6]. Nevertheless, autoreactive antibody production needs to be supported by helper $\mathrm{T}(\mathrm{Th})$ cells with certain subtype differentiations. In autoimmune disorders, as in MG, the role of type-1 Th (Th1) cells, the Th1/Th2 balance and the amount of regulatory $T$ (Treg) cells are well-acknowledged [7-11]. Th17 subtype emerged as a critical promoter of inflammation that can contribute to onset and exacerbation of autoimmune reactions $[12,13]$. On the other hand, the role of Th17 in MG is not well-established and no data is available if Th17 cells present in the MG thymus tissue.

Not only B and T cells but also innate lymphoid cells, e.g. natural killer (NK) cells, are associated with MG [14]. NK cells possess a capacity to modulate the actions of both Th17 cells and B lymphocytes [14-17]. Under certain inflammatory conditions, interferon (IFN)- $\gamma$ produced by NK cells was implicated in autoimmune pathology and these cells were also determined to participate in the development of experimental MG $[18,19]$. Intriguingly, NK cell-mediated immunity was also identified as a critical modulator of Th17 responses [16]. Th17-polarizing conditions are hampered because of the IFN- $\gamma$ secreted by NK cells [16,17]; thus, NK cells have been indirectly implicated in maintenance of tolerance and suppression of central nervous system autoimmunity. Nevertheless, even though the role of thymus is obvious in the disease etiopathology, to date, the presence of NK and Th17 cells was not reported in the MG thymus.

\section{MATERIALS AND METHODS}

\section{Patient and Control Groups}

Thymus tissues excised from MG patients $(n=49)$ with anti-AChR antibody positivity were used in this study (Table 1). In addition to the freshly collected specimens $(n=12), 37$ formalin-fixed, paraffin-embedded tissue samples were obtained from pathology archives. Tissue sections were stained with hematoxylin-eosin and evaluated histopathologically under conventional light microscopy. Histopathological classification of the thymic lesions (thymoma, $n=19$; follicular hyperplasia, $n=11$ and normal thymus, $\mathrm{n}=19$ ) was based on the World Health Organization criteria. Thymus tissues collected with thyroid or parathyroid surgeries (for parathyroid adenoma and/or goiter) were used as a non-MG control group ( $n=11)$. Majority of the patients $(n=37)$ underwent various immune modulatory therapies whereas 12 patients were naïve before thymectomy (Table 1). This study was approved by local ethics committee (GO 13/34-32).

Table 1. The demographic, clinical and laboratory findings of MG patients

\begin{tabular}{|l|c|c|c|}
\hline Thymus pathology & Thymoma(n=19) & Follicular hyperplasia( $\mathbf{n}=\mathbf{1 1})$ & Normal thymus(n=19) \\
\hline $\begin{array}{l}\text { Age of onset (years) } \\
\text { Mean } \pm \text { SD (min-max) }\end{array}$ & $46 \pm 14(22-73)$ & $30 \pm 13(17-56)$ & $32 \pm 13(13-69)$ \\
\hline $\begin{array}{l}\text { Age of thymectomy (years) } \\
\text { Mean } \pm \text { SD (min-max) }\end{array}$ & $47 \pm 14(22-74)$ & $31 \pm 13(19-57)$ & $35 \pm 13(15-69)$ \\
\hline Male/ Female & $7 / 12$ & $2 / 9$ & $6 / 13$ \\
\hline Antibody level & $63 \pm 93(2-395)$ & $171 \pm 213(2-640)$ & $123 \pm 372(0.6-1600)$ \\
Mean \pm SD (min-max) & $6 / 13$ & $0 / 11$ & $2 / 17$ \\
\hline Initial symptoms & & & 15 \\
Ocular/Generalized & 8 & 8 & 1 \\
\hline Treatment before thymectomy* & 1 & 1 & 1 \\
\hline CS & 0 & 0 & 4 \\
AZA & 4 & 3 & 2 \\
MM & 5 & 2 & 3 \\
\hline IVlg & 7 & 2 & \\
PE & & & \\
No treatment & & & \\
\hline
\end{tabular}


AZA, azathioprine; CS, corticosteroids; IVIg, intravenous immunoglobulin; $\mathrm{MM}$, micophenolate mofetil; PE, plasma exchange; SD, standard deviation

*Some patients were on more than one agent.

\section{Flow Cytometric Immunophenotyping}

Thymus tissue was chopped and subjected to enzymatic digestion with collagenase type II $(0.075 \%$, Sigma, St. Louis, Missouri, USA) for $4 \mathrm{~h}$; then, the digestion mixture was passed through a $40 \mu$ m nylon mesh (Fisher Scientific, Hampton, New Hampshire, USA). The cells layered at the interphase of Ficoll 1077 (Sigma) density fraction were directly collected and washed. The thymocytes were labeled with monoclonal antibodies anti-human-CD2 (S5.2), -CD3 (SK3), -CD56 (NCAM16.2 or HCD56), -CD16 (B73.1), -NKG2D (1D11), -NKp46 (9-E2) (Becton Dickinson, San Jose, CA, USA) and -IL-23R (218213) (LifeSpan Biosciences, Seattle, Washington, USA). The percentage of positive cells was gated according to the staining with isotype-matched antibody controls. Immunophenotyping was performed on a FACSAria II flow cytometer (Becton Dickinson).

\section{Immunohistochemistry (IHC)}

Sections $(4 \mu \mathrm{m})$ from formaldehyde-fixed and paraffin-embedded tissue materials were deparaffinized, rehydrated and incubated with primary antibodies against human IL-21R (Thermo Scientific, Waltham, Massachusetts, USA, 1/300), IL-23R (1/200) and ROR- $\gamma$ (1/200) (LifeSpan). Antibody binding was determined by using Bond Polymer Refine Detection kit (Leica Biosystems, Newcastle Upon Tyne, UK). Harris's hematoxylin was used as counterstain. The IHC results was analyzed according to the prevalence of positively stained cells and qualitatively scored as "no," "slight," and "apparent."

\section{Statistical Methods}

Statistical analyses were done using IBM SPSS 21 software (IBM Corp. Released 2012. IBM SPSS Statistics for Windows, Version 21.0. Armonk, NY: IBM Corp). Descriptive statistics were presented as number and percentage for qualitative variables and as mean \pm standard deviation (SD) for numerical variables. Difference between two groups (patient and control) in terms of age was assessed by independent samples t-test, whereas the difference between four groups was evaluated by one-way ANOVA. Comparison of genders between the groups was done using Chi-square test. Difference between two groups was assessed by Mann-Whitney $U$ test and the difference between four groups was assessed by Kruskal Wallis test for non-normally distributed variables. Pairwise comparisons were done by Dunn test. Relations between variables were investigated by Spearman's rank correlation coefficient. A P value $\leq 0.05$ was considered statistically significant.

\section{RESULTS}

\section{NK Cells and IL-23R+ T Cells Present in MG Thymus}

The thymus has been acknowledged as a niche for $T$ cell development and the presence of differentiated T cells or NK cells are rarely reported [20]. Since the interaction between NK cells and Th17 cells has been underlined in autoimmune disorders [16,17], we asked whether these cells are found in the MG thymus. By using a flow cytometric immunophenotyping strategy, NK cells were detected (CD3-CD16/56+, range $0.5-15.2 \%)$ in amongst the leukocytes isolated from the thymus tissue (Figure $1 \mathrm{~A}$ and $\mathrm{B}$ ). Then, these cells were gated and their activation status was analyzed with NKG2D and NKp46 expression (Figure 1A). Of 12 patients analyzed, five had no expression of NKG2D or NKp46 on NK cells; these were especially that of the thymus specimens harboring scant amount of NK cells (Figure 1C). Alternatively, a group of patients possessed NK cells with an activated phenotype. NKG2D was more frequently detected (NKG2D+, range 11.8-68.6\%) than NKp46 (NKp46+, range 3.8-51.9\%) (Figure 1C) whereas majority of the NKG2D+ cells co-expressed the NKp46 cytotoxicity receptor (Figure 1A). 


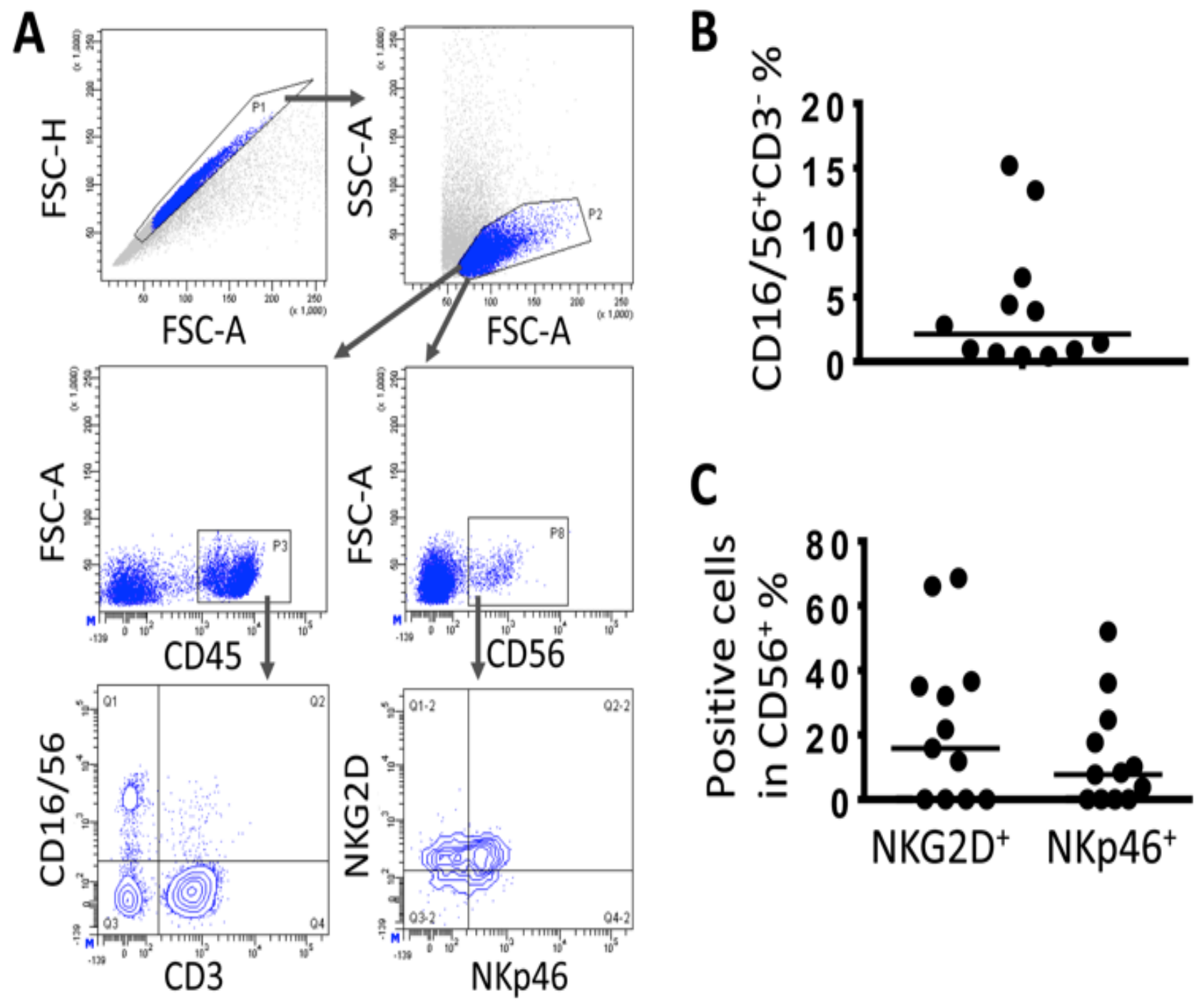

Figure 1. The presence of NK cells in the thymus tissues excised from MG patients was studied by flow cytometry. A) Gating strategy used for the immunophenotype analysis of CD45+CD3-CD16/56+ NK cells and NKG2D+ and/or NKp46+ subpopulations in CD56+ NK cells are shown. The percentage distribution of (B) CD45+CD3-CD16/56+ NK cells and (C) NKG2D+ and/or NKp46+ NK subpopulations in the thymocyte suspensions prepared from each MG patient is given. In the dot-plots, the horizontal lines indicate the mean value.

Since the thymus is the primary immune organ where $T$ cells gain capacity to recognize antigens, thus, the common $T$ cell marker CD3 is modulated on all thymocytes [21]. Therefore, both CD2 and CD3 markers were used to determine thymocytes. Except in one patient, approximately all thymocytes were positive for CD2 (CD2+, 88.92 $\pm 15.85 \%)$. Expectedly, the percentage of $\mathrm{CD} 3+$ cells remained limited (CD3+, $59.98 \pm 18.83 \%$ ) (Figure $2 A$ and $C$ ). As a surface marker associated with Th17 cells, the receptor for IL-23 (IL23R) was found on a subpopulation of $\mathrm{CD} 3+\mathrm{T}$ cells
(CD3+IL-23R+, range 3.7-33.45\%) (Figure 2B and D). These cells were regarded as evidence for the presence of Th17 subset in the MG thymus. Moreover, a positive correlation ( $r=0.68)$ was determined between NK cells and IL-23R+ T cells in the MG thymus (Figure 3). Additionally, in terms of NK or T cells, there was no difference amongst the MG patients with different thymus histopathologies, i.e. thymoma, follicular hyperplasia, and normal histology (data not shown). 
A

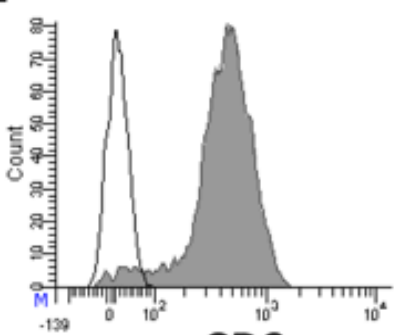

CD2

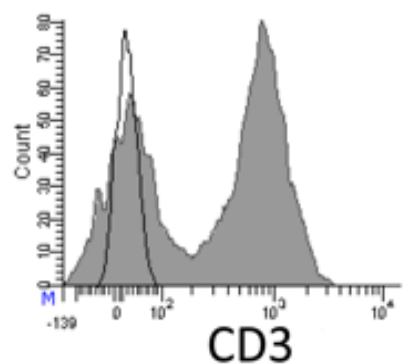

B
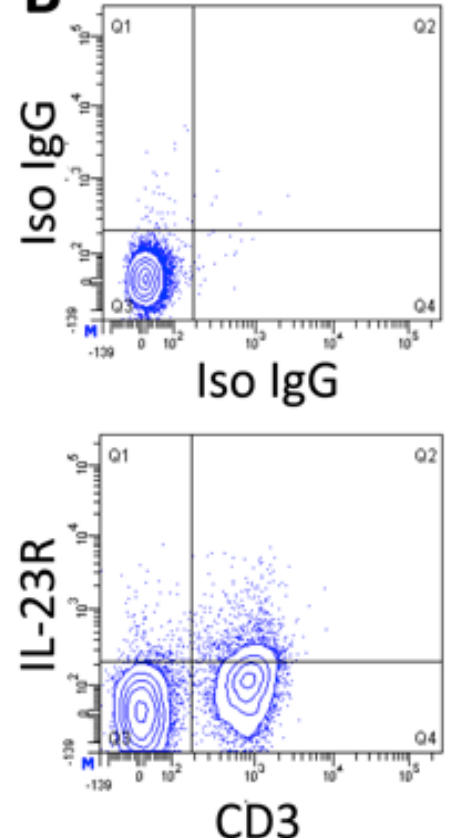

C

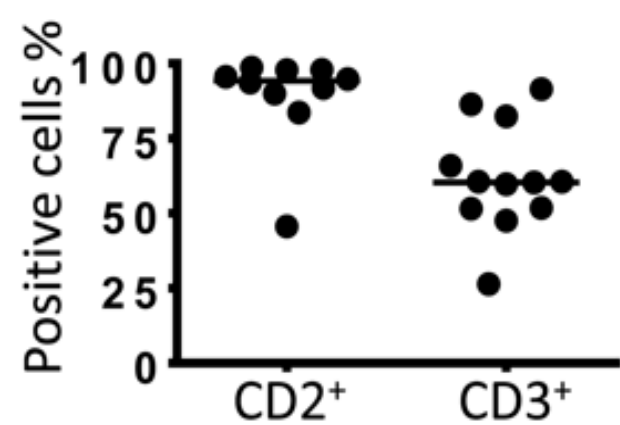

D

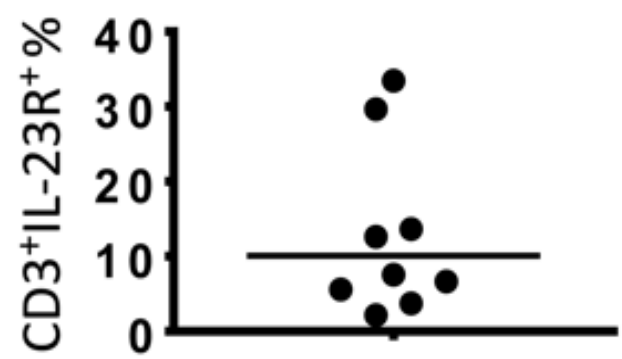

Figure 2. The amount of $C D 2+$ or $C D 3+$ thymocytes and IL-23R positivity in the MG thymus. Representative flow cytometry histograms (A) for the CD2 or CD3 positive thymocyte populations (empty histogram, isotype control staining; filled histogram, specific antibody staining) and (B) for the expression of IL-23R on the CD3+ cells are given. Upper and lower scatter dot-plots represent the isotype control staining and the specific antibody staining, respectively. The percentage distribution of (C) the cells positive for CD2 or CD3, and (D) IL-23R+ subpopulation in CD3+ thymocytes is given. The horizontal lines indicate the mean value.

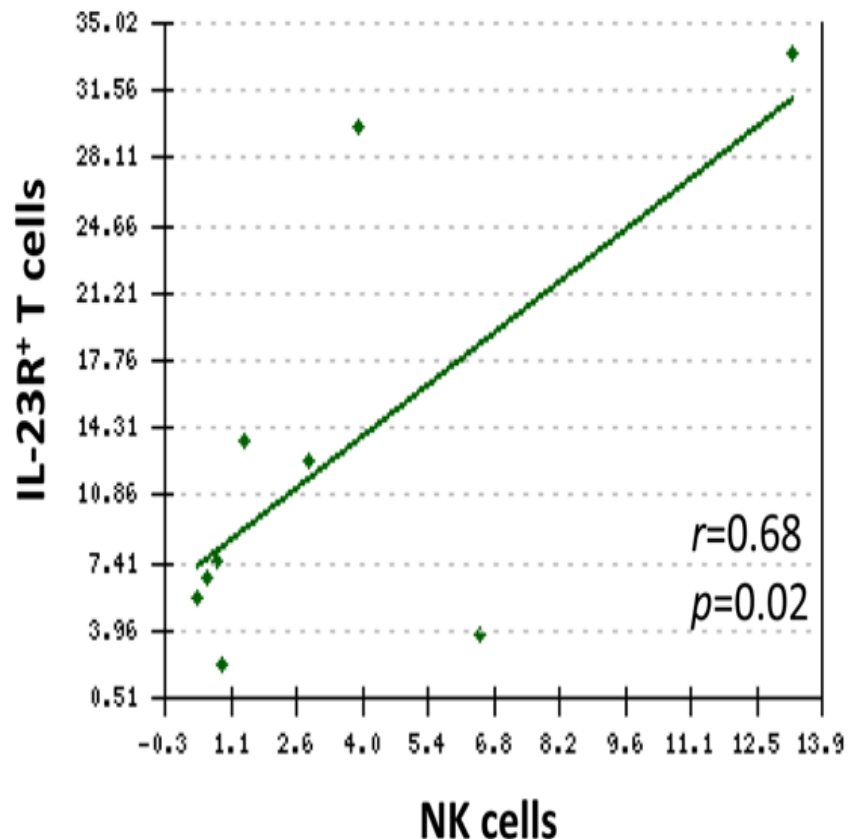

Figure 3. The correlation analysis between the percentage distribution of IL-23R+CD3+ thymocytes and CD56+ NK cells in the thymus samples obtained from MG patients. 


\section{Validation of Th17-Associated Markers in The MG Thymus}

Expression of IL-23R, IL-21R, and ROR- $\gamma$ as Th17related markers [22-25] was evaluated in the thymus tissues from MG patients and the non-MG control patients. In general, the percentage distribution of specimens with a slight IL-23R and ROR- $\gamma$ expression was significantly lower in MG patients compared to that of the control group (Figure 4A). Approximately $20 \%$ of the MG patients possessed identifiable thymocytes positive for IL-23R or IL-21R whereas ROR- $\gamma$ was only detected in a minor group of patients ( $<10 \%)$ (Figure $4 A$ ). Accordingly, only the thymus tissues with a prominent expression of IL-23R, IL-21R and/or ROR- $\gamma$ markers were considered to harbor a Th17 population (Figure 4C).

When MG thymus tissues were categorized according to histopathological subtypes thymomas significantly displayed less apparent expression of all three markers $(P<0.05)$. A group of $M G$ patients with follicular hyperplasia or normal thymic histology had apparent staining for IL-23R or IL-21R. In the thymus with normal histology, these markers tend to be more widely expressed amongst the patients (Figure 4B). A positive correlation was observed between IL-23R and ROR- $\gamma$ in the MG thymus with normal histology and in thymoma $(r=0.58, P<0.05$ and $r=0.56, P<0.05$, respectively). In addition, IL-21R and IL-23R levels were in correlation $(r=0.57, P<0.05)$ in thymomas.

On the other hand, none of the clinical parameters and patient characteristics (anti-AChR antibody titers, age, sex, and therapy received prior to thymectomy) were associated with the presence or amount of either NK or Th17-related markers (data not shown).
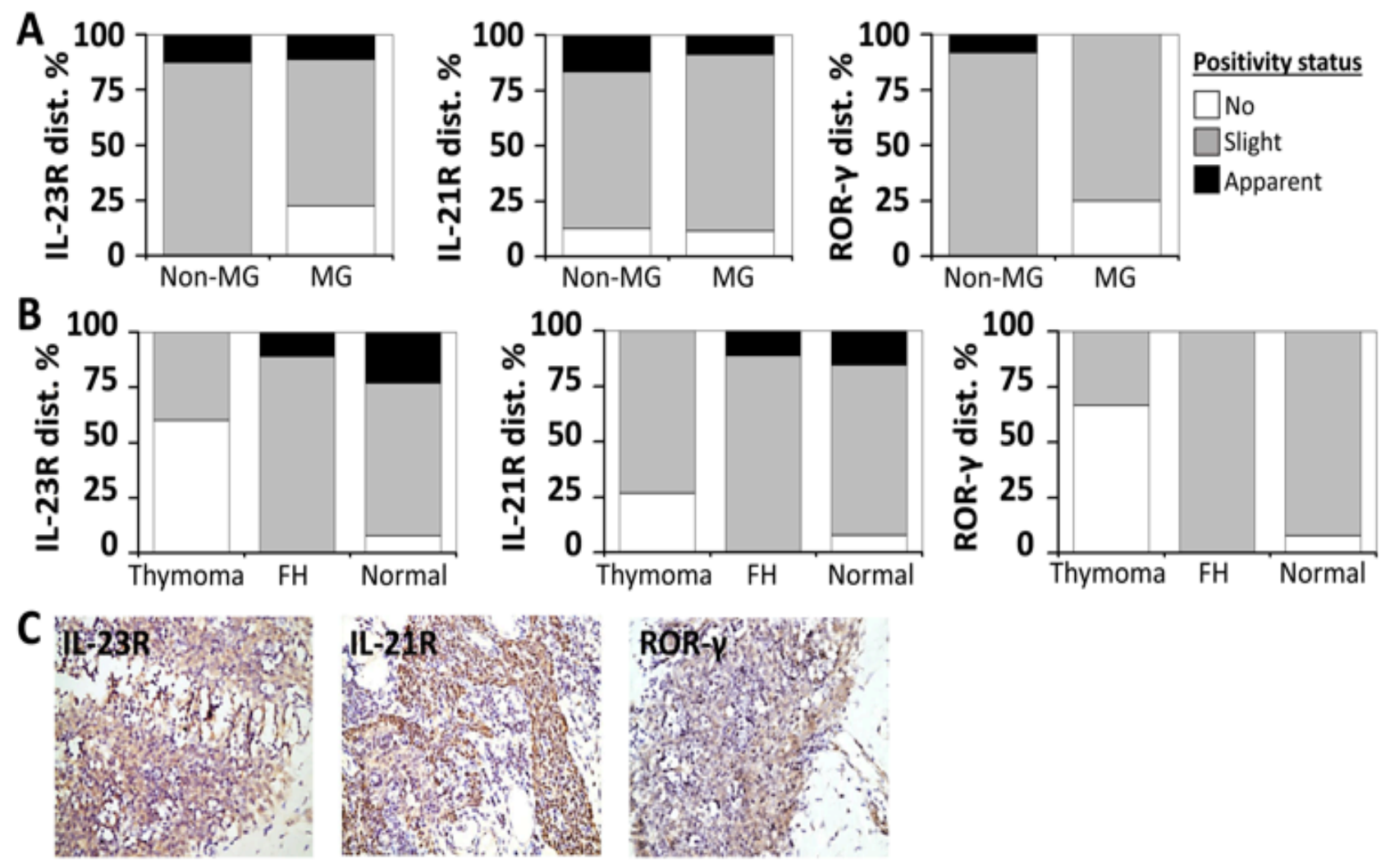

Figure 4. Percentage distribution of MG patients' thymectomy specimens according to IL-21R, IL-23R, and ROR-y expression levels. The paraffin embedded tissues were obtained from pathology archives and stained by immunohistochemistry. A) Comparison between the percentage distribution of non-MG and MG patients according to IL-23R, IL-21R, and ROR- $\gamma$ positivity in thymus specimens. The patient samples were grouped according to a qualitative (no, slight, apparent) staining rank. B) Comparison between the percentage distribution of MG patients with thymoma, follicular hyperplasia (FH) or normal thymus histopathology according to IL-23R, IL-21R, and ROR- $\gamma$ positivity. The patient samples were grouped according to a qualitative (no, slight, apparent) staining rank. C) Representative micrographs of MG thymus samples with an apparent histochemical staining for IL-23R, IL-21R, and ROR- $\gamma$ can be seen. 


\section{DISCUSSION}

Even though the role of thymus in anti-AChR antibody positive MG is undoubted, which is basically due to the clinical benefit obtained after removal of this organ [1,2], alterations in its immunological composition is not widely studied. Here, the existence of NK cells and Th17 cells are reported in the thymus of anti-AChR antibody positive MG patients. The presence of these immune cell types in the thymus may have critical implications since Th17-mediated autoimmune responses can be modulated by NK cells $[15-18,26]$.

Even though NK cells are known with their cytotoxic and proinflammatory functions, they have also been implicated in the maintenance of tolerance $[27,28]$. NK cells contribute to the first line of defense against viral infections and tumor cells $[19,28]$. Direct killing of the infected or transformed cells and secretion of IFN- $\gamma$ mediate differentiation of Th1 cells $[19,29]$. IFN- $\gamma$ not only promotes Th1-mediated anti-viral or anti-tumor immunity but also interferes with the differentiation of other T helper subtypes, including Th17 cells [29]. Therefore, in MG, especially which manifest with hyperplasia or thymoma, the presence of NK cells in the thymic microenvironment is of interest. On the other hand, the thymus plays a central role in MG autoimmunity and previous studies indicated a possible role for Th17 cells in MG $[12,30]$. IL-21R, IL-23R and thymic ROR- $\gamma$ (ROR- $\gamma \mathrm{t}$ ) are amongst the reliable Th17 markers even though their expression is not restricted to these cells $[13,31,32]$. IL-21R is expressed particularly in T cells, $B$ cells and NK cells while IL-23R is found on the surface of memory T cells, NK cells, macrophages, dendritic cells, and monocytes [31]. Therefore, the thymocytes (i.e., the lymphocytic cells in the thymus) that were positively stained with IL-21R and IL-23R can be either NK or Th17 cells. The maintenance of Th17 subtype and the escalation of NK functions depends on IL-21 and IL-23 cytokines $[13,22-25,33]$. These cytokines may have a potential to be involved in MG immunopathology in thymus. Previously, expression of IL-6, IL-17A, IL-17F,
IL-17C, and TGF- $\beta 1$ was detected in MG thymus as Th17-associated cytokines [34]. The transcription factors ROR- $\gamma$ and RORa are implicated in Th17 differentiation but in the thymus, ROR- $y \mathrm{t}$ is also expressed by the lymphoid tissue-inducing cells $[22,32]$. Therefore, not underscoring the asset of IL-21R, IL-23R, and ROR- $\gamma$ as pivotal markers associated with Th17 subset, functional assays would be critical to define these cells in the thymus.

Previously, MG patients with high anti-AChR antibody titers were identified with increased amounts of IL-17 and CD4+IL-17+ cells.30,35 In our study, there was no association between the thymic Th17 cells and the serum anti-AChR antibody levels and this observation was independent of the patients' clinical history and therapy received. Therefore, Th17 subset that populate the thymus may not directly influence the autoreactive antibody production in MG. On the other hand, in the thymus of non-MG patients with parathyroid adenoma or goiter, the lymphocytic cells displayed similar IL-21R, IL-23R, and ROR- $\gamma$ expression with MG thymocytes. Nevertheless, the effect of thyroid pathologies on neighboring thymus tissue cannot be ruled out in this non-MG control group. Intriguingly, the thymocytes with IL-21R, IL-23R, and ROR- $\gamma$ positivity were significantly reduced in the MG thymus with thymoma histopathology. In contrast, increased numbers of Th17 cells was found in the circulation of MG patients with thymoma [30]. Therefore, discrepancies in the regulation of immune responses among the histopathological subtypes of MG thymus and between the local thymic tissue and the peripheral blood may be argued $[5,6,30]$. Nevertheless, the presence of other immune cell subsets, e.g. Treg cells or myeloid cells $[9,36]$, and their functional status must be simultaneously analyzed to better understand the immune regulation in MG thymus.

This study was limited by the analysis of thymocytes only. Thus, new prospective functional studies comparing the lymphocyte subset in peripheral blood and thymus are needed to better understand the interaction of these cells and their role in MG pathogenesis. Moreover, functional assays on freshly isolated Th17 cells and NK cells from MG thymus would increase the insights on the contribution of these cells to immune 
dysregulation in MG. The interaction between NK ACKNOWLEDGMENTS

and Th17 cells is a rather new concept and its impact

can be critical in tolerance and autoimmunity. Even though isolation of both cell types from the same

microenvironment does not readily indicate a direct cooperation between these cell types, our data indicate the plausibility to investigate the cross-regulation of NK and Th17 cells in MG.

This study is supported by Ankara Society of Neuroimmunology and Hacettepe University Scientific Research Project (BAP: 259). The authors thank Z. Birinci for technical assistance.

\section{CONFUCT OF INTEREST}

None declared. 
[1] Vincent A, Palace J, Hilton-Jones D. Myasthenia gravis. Lancet. 2001; 357: 2122-2128.

[2] Berrih-Aknin S, Le Panse R. Myasthenia gravis: A comprehensive review of immune dysregulation and etiological mechanisms. J Autoimmun. 2014; 52: 90-100.

[3] Berrih-Aknin S, Morel E, Raimond F, et al. The role of the thymus in myasthenia gravis: immunohistological and immunological studies in 115 cases. Ann N Y Acad Sci. 1987; 505: 50-70. [4] Schluep M, Willcox N, Vincent A, et al. Acetylcholine receptors in human thymic myoid cells in situ: an immunohistological study. Ann Neurol. 1987; 22: 212-222.

[5] Strobel P, Rosenwald A, Beyersdorf N, et al. Selective loss of regulatory T cells in thymomas. Ann Neurol. 2004; 56: 901-904. [6] Kadota Y, Okumura M, Miyoshi S, et al. Altered T cell development in human thymoma is related to impairment of $\mathrm{MHC}$ class II transactivator expression induced by interferon-gamma (IFN-gamma). Clin Exp İmmunol. 2000; 121: 59-68.

[7] Baecher-Allan C, Hafler DA. Human regulatory T cells and their role in autoimmune disease. Immunol Rev. 2006; 212: 203-216.

[8] Wang HB, Shi FD, Li H, et al. Role for interferon-gamma in rat strains with different susceptibility to experimental autoimmune myasthenia gravis. Clin Immunol. 2000; 95: 156-162.

[9] Balandina A, Lecart S, Dartevelle P, et al. Functional defect of regulatory $\mathrm{CD} 4(+) \mathrm{CD} 25+\mathrm{T}$ cells in the thymus of patients with autoimmune myasthenia gravis. Blood. 2005; 105: 735-741.

[10] Luther C, Poeschel S, Varga $M$, et al. Decreased frequency of intrathymic regulatory T cells in patients with myasthenia-associated thymoma. J Neuroimmunol. 2005; 164: 124-128. [11] Fattorossi A, Battaglia A, Buzzonetti A, et al. Thymopoiesis, regulatory $T$ cells, and TCRVbeta expression in thymoma with and without myasthenia gravis, and modulatory effects of steroid therapy. J Clin Immunol. 2008; 28: 194-206.

[12] Mu L, Sun B, Kong Q, et al. Disequilibrium of T helper type 1, 2 and 17 cells and regulatory T cells during the development of experimental autoimmune myasthenia gravis. Immunology. 2009; 128: e826-836

[13] Ouyang W, Kolls JK, Zheng Y. The biological functions of T helper 17 cell effector cytokines in inflammation. Immunity. 2008; 28: 454-467.

[14] Shi FD, Wang HB, Li H, et al. Natural killer cells determine the outcome of B cell-mediated autoimmunity. Nat Immunol. 2000; 1(3): 245-51.

[15] Hao J, Liu R, Piao W, et al. Central nervous system (CNS)resident natural killer cells suppress Th17 responses and CNS autoimmune pathology. J Exp Med. 2010; 207(9):1907-21.

[16] Wu W, Shi S, Ljunggren HG, et al. NK cells inhibit T-betdeficient, autoreactive Th17 cells. Scand J Immunol. 2012; 76(6): 559-66.

[17] Fu B, Li X, Sun R, et al. Natural killer cells promote immune tolerance by regulating inflammatory $\mathrm{TH} 17$ cells at the human maternal-fetal interface. PNAS. 2013; 110(3): E231-40.

[18] Suzuki Y, Onodera H, Tago $H$, et al. Altered populations of natural killer cell and natural killer T cell subclasses in myasthenia gravis. J Neuroimmunol. 2005; 167(1-2): 186- 9.
[19] Kott E, Hahn T, Huberman M, et al. Interferon system and natural killer cell activity in myasthenia gravis. Q J Med. 1990; 76(281): 951-60.

[20] Spits H, Lanier LL, Phillips JH. Development of human T and natural killer cells. Blood. 1995; 85(10): 2654-70.

[21] Dave VP. Hierarchical role of CD3 chains in thymocyte development. Immunol Rev. 2009; 232(1): 22-33.

[22] Dong C. TH17 cells in development: an updated view of their molecular identity and genetic programming. Nat Rev Immunol. 2008; 8: 337-348.

[23] Korn T, Bettelli E, Gao W, et al. IL-21 initiates an alternative pathway to induce proinflammatory $\mathrm{T}(\mathrm{H}) 17$ cells. Nature. 2007;448: 484-487.

[24] Nurieva R, Yang XO, Martinez G, et al. Essential autocrine regulation by IL-21 in the generation of inflammatory $T$ cells. Nature. 2007; 448: 480-483.

[25] Zhou L, Ivanov II, Spolski R, et al. IL-6 programs T(H)-17 cell differentiation by promoting sequential engagement of the IL21 and IL-23 pathways. Nat Immunol. 2007; 8: 967-974.

[26] Chien PJ, Yeh JH, Chiu HC, et al. Inhibition of peripheral blood natural killer cell cytotoxicity in patients with myasthenia gravis treated with plasmapheresis. Eur J Neurol. 2011; 18(11): 1350-7.

[27] Jeager BN, Vivier E. Natural killer cell tolerance: control by self or self-control? Cold Spring Harb Perspect Biol. 2012; 4(3). pii: a007229.

[28] Shifrin N, Raulet DH, Ardolino M. NK cell self tolerance, responsiveness and missing self recognition. Semin Immunol. 2014; 26(2): 138-44.

[29] Luckheeram RV, Zhou R, Verma AD, et al. CD4+T cells: Differentiation and functions. Clin Dev Immunol. 2012; 2012: 925135.

[30] Wang Z, Wang W, Chen Y, et al. T helper type 17 cells expand in patients with myasthenia-associated thymoma. Scand J Immunol. 2012; 76: 54-61.

[31] Tan ZY, Bealgey KW, Fang Y, et al. Interleukin-23: immunological roles and clinical implications. Int J Biochem Cell Biol. 2009; 41: 733-735.

[32] Eberl G, Marmon S, Sunshine MJ, et al. An essential function for the nuclear receptor RORgamma(t) in the generation of fetal lymphoid tissue inducer cells. Nat Immunol. 2004; 5: 64-73. [33]Cacalano NA. Regulation of Natural Killer Cell Function by STAT3. Front Immunol. 2016; 7: 128.

[34] Cordiglieri C, Marolda R, Franzi S, et al. Innate immunity in myasthenia gravis thymus: pathogenic effects of Toll-like receptor 4 signaling on autoimmunity. J Autoimmun. 2014; 52: 74-89.

[35] Roche JC, Capablo JL, Larrad L, et al. Increased serum interleukin-17 levels in patients with myasthenia gravis. Muscle Nerve. 2011; 44: 278-280.

[36] Fan X, Zhang H, Cheng Y, et al. Double Roles of Macrophages in Human Neuroimmune Diseases and Their Animal Models. Mediators Inflamm. 2016; 2016:8489251. 